\title{
O CENTENÁRIO DA MOLÉCULA DE BOHR
}

\author{
Carlos A. L. Filgueiras* e João P. Braga \\ Departamento de Química, Universidade Federal de Minas Gerais, 31270-901 Belo Horizonte - MG, Brasil \\ Nelson H. T. Lemes \\ Instituto de Química, Universidade Federal de Alfenas, 37130-000 Alfenas - MG, Brasil
}

Recebido em 24/6/13; aceito em 1/7/13; publicado na web em 19/7/13

\begin{abstract}
THE CENTENNIAL OF BOHR'S MOLECULE. A hundred years ago, a twenty-eight year old Danish scientist published a series of three papers in which electron motion was quantized. The Bohr atomic model is surely known by every chemistry student. Nevertheless in this same 1913 trilogy, Bohr studied atoms with several electrons as well as molecules. Chemistry students, in general, are not aware of the Bohr molecule. The present paper aims at rescuing this important classical model. A review of the Bohr atomic model for both one and several electrons is discussed, together with a theoretical presentation of the Bohr molecule.
\end{abstract}

Keywords: Niels Bohr; Bohr's atoms and molecules; the constitution of atoms and molecules.

\section{INTRODUÇÃO}

Todo aluno de química conhece o átomo de Bohr. O assunto é abordado no ensino médio, nas disciplinas universitárias de Química Geral e de Química Quântica. Entretanto, ao perguntar a esse mesmo aluno se ele tem conhecimento da molécula de Bohr a resposta é, quase sempre, negativa. Essa pergunta foi feita na sala de aula de quatro disciplinas de Físico-Química nas respectivas universidades dos autores e, na verdade, a resposta foi negativa em todos os casos. Portanto, o aluno de química não sabe o que é a molécula de Bohr, mas tem conhecimento do átomo de Bohr. É também bastante curioso que o título do trabalho original de Bohr é: "Sobre a constituição de átomos e moléculas". A conclusão é simples, são poucos os que realmente recorrem ao texto original para entender o trabalho de Bohr. ${ }^{1,2}$

O trabalho original de Bohr é dividido em três partes. Na parte I é apresentado o que se conhece por átomo de Bohr. Na segunda parte, Bohr explora átomos multieletrônicos e antecipa o conceito de carga atômica efetiva. Na parte III é apresentada a molécula de $\mathrm{H}_{2}$, que, por analogia com o átomo de Bohr deve ser denominada de molécula de Bohr. Para deixar este ponto claro deve-se prestar atenção aos títulos e subtítulos desses trabalhos:

1. Sobre a constituição de átomos e moléculas. Parte I - Ligação de elétrons por núcleos positivos;

2. Sobre a constituição de átomos e moléculas. Parte II - Sistemas que contêm um só núcleo.

3. Sobre a constituição de átomos e moléculas. Parte III - Sistemas que contêm vários núcleos.

Os pontos principais das partes II e III serão aqui apresentados, com descrição inicial da parte I, pois nessa parte são introduzidas as equações básicas. O modelo molecular de Bohr, além de sua importância histórica, ${ }^{3}$ oferece também aspectos importantes para o ensino de ligação química e a superfície de energia potencial. Espera-se que o presente trabalho motive o estudo da molécula de Bohr e que esse modelo seja explorado no curso de química, a exemplo do que já é feito com o átomo de Bohr.

\section{O ÁTOMO DE BOHR}

As ideias de Bohr foram desenvolvidas antes da mecânica quântica, num período após a descoberta de Planck e antes da equação de Schrödinger. Ideias clássicas, como o movimento orbital do elétron, eram permitidas nesse período. A quantização da ação era o ponto importante desse período, pois a constante de Planck tem unidade de ação, o quantum de ação. Assumia-se que propriedades periódicas eram proporcionais à constante de Planck. A teoria dessa época, que envolve uma mistura de mecânica clássica e quântica pela quantização da ação, é hoje conhecida como a mecânica quântica antiga. Niels Bohr inaugurou esse período.

As ideias de Bohr foram baseadas nos trabalhos de Planck, Rutherford e na espectroscopia atômica. Nas Figuras 1 e 2 são apresentadas duas fotografias de Niels Bohr. A primeira delas, com Max Planck, foi tirada em 1930 no Instituto Niels Bohr de Copenhagen. Na segunda das fotografias, de 1933, Niels Bohr caminha com Ernest Rutherford durante o Congresso Solvay de 1933 em Bruxelas.

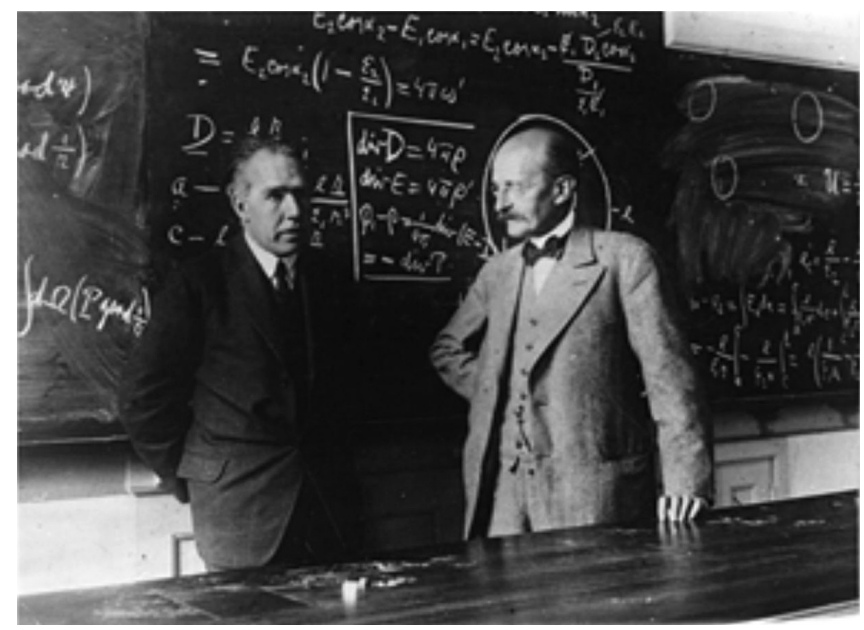

Figura 1. Niels Bohr e Max Planck em 1930 no Instituto Niels Bohr. Foto gentilmente cedida pelo Arquivo Bohr, Copenhagen

Dentro de um contexto da mecânica quântica antiga, considerase um elétron girando em torno do núcleo, conforme a Figura 3, representando o átomo de Bohr. Se o núcleo tem carga $Z$ pode-se igualar a força centrífuga com a eletrostática para fornecer, $\frac{m v^{2}}{r}=\frac{Z e^{2}}{r^{2}}$. 


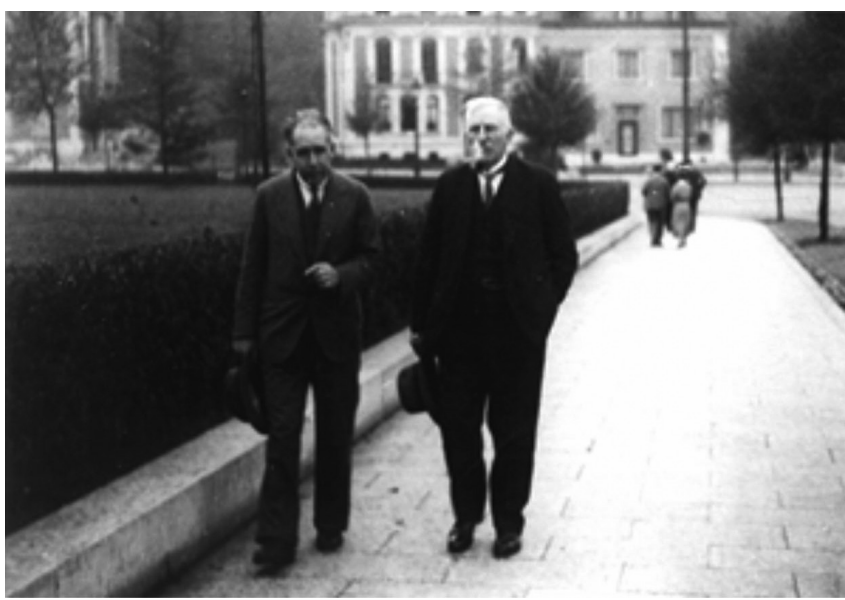

Figura 2. Niels Bohr em 1933 com seu mentor, Ernest Rutherford. Os dois se encontraram no Congresso Solvay em Bruxelas. Foto gentilmente cedida pelo Arquivo Bohr, Copenhagen

Por conseguinte, a energia cinética será, $E_{\mathrm{c}}=\frac{m v^{2}}{2}=\frac{Z e^{2}}{2 r}$. A energia potencial da interação do elétron com o núcleo é dada por, $E_{\mathrm{p}}=-\frac{Z e^{2}}{r}$, indicando que $E=E_{\mathrm{c}}+E_{\mathrm{p}}=-\frac{Z e^{2}}{2 r}$. A relação $-2 E_{\mathrm{c}}=E_{\mathrm{p}}$ é consequência do teorema do virial na mecânica clássica e como tal foi explorada por Bohr em seu trabalho. ${ }^{2} \mathrm{O}$ equilíbrio de forças, $\frac{m v^{2}}{r}=\frac{Z e^{2}}{r^{2}}$, pode ser reescrito na forma $m^{2} v^{2} r^{2}=m r Z e^{2}$ para se usar a quantização do momento angular, $L=m v r=n \hbar$. Portanto, $n^{2} \hbar^{2}=m r Z e^{2}$ ou $r=\frac{n^{2} \hbar^{2}}{m Z e^{2}}$, em que $r$ é conhecido como raio de Bohr.

As equações básicas da parte I são, ${ }^{1,3}$

$$
\begin{aligned}
& r=\frac{n^{2} \hbar^{2}}{m Z e^{2}} \\
& E=-\frac{m Z^{2} e^{4}}{2 n^{2} \hbar^{2}} \\
& v=\frac{1}{2 \pi} \frac{m Z^{2} e^{4}}{n^{3} \hbar^{3}}=\frac{4 \pi^{2} m Z^{2} e^{4}}{n^{3} h^{3}}
\end{aligned}
$$

A frequência é estabelecida pelo raio de Bohr e energia, pois usando $E_{\mathrm{c}}=-E$ retira-se $v$ e pode-se calcular a frequência angular, $\omega=2 \pi v=\frac{v}{r}$. Essas equações equivalem às equações (3) da página 100 da tradução do trabalho de Bohr para o português. ${ }^{1}$ Somente essas equações são apresentadas ao aluno de química. Elas constituem a base teórica do modelo do átomo de Bohr.

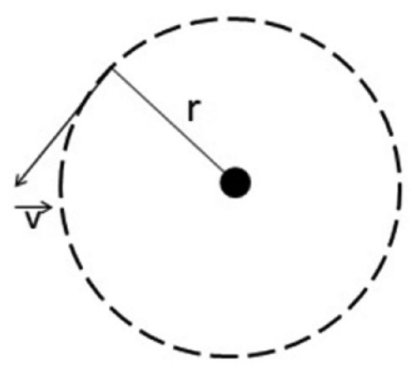

Figura 3. Modelo atômico de Bohr

\section{ÁTOMOS COM DOIS ELÉTRONS}

Ainda na parte I, Bohr descreve átomos de muitos elétrons, mas concentra-se no assunto na parte II da trilogia. Bohr descreve a estabilidade de um sistema com um só núcleo, mas com vários elétrons numa única camada. Primeiramente reescreve as equações (1) mudando o número atômico $Z$ para outro símbolo, que ele denotou por $F$. Nesse ponto iguala a força centrífuga a $\frac{e^{2}}{a^{2}} F$,

$$
\frac{m v^{2}}{a}=\frac{e^{2}}{a^{2}} F
$$

antecipando assim o conceito de carga atômica efetiva. Por um raciocínio completamente análogo ao de átomos de um elétron obtém as relações,

$$
\begin{aligned}
& r=\frac{n^{2} \hbar^{2}}{m F e^{2}} \\
& E=-\frac{m F^{2} e^{4}}{2 n^{2} \hbar^{2}} \\
& v=\frac{1}{2 \pi} \frac{m F^{2} e^{4}}{n^{3} \hbar^{3}}=\frac{4 \pi^{2} m F^{2} e^{4}}{n^{3} h^{3}}
\end{aligned}
$$

equivalentes às equações (1). Estas são as equações (1)-(3) da parte II, página 136 da versão em português. ${ }^{1}$

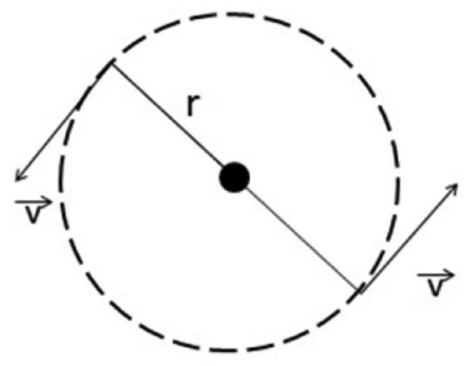

Figura 4. Modelo atômico de Bohr para o átomo de hélio

Uma ilustração inicial para átomos de dois elétrons, com número atômico $Z$, pode ser feita igualando-se a força centrífuga de uma órbita circular de raio $a$ com as forças elétron-elétron e elétron-núcleo, conforme Figura 4. Para elétrons diametralmente opostos com raio $a$, tem-se, $\frac{m v^{2}}{a}=\frac{Z e^{2}}{a^{2}}-\frac{e^{2}}{(2 a)^{2}}$, ou,

$$
\frac{m v^{2}}{a}=\left(Z-\frac{1}{4}\right) \frac{e^{2}}{a^{2}}
$$

Logo, por comparação com (2) retira-se $F=Z-\frac{1}{4}$. Para o átomo de hélio tem-se,

$$
F=Z-\frac{1}{4}=2-\frac{1}{4}=1,75
$$

em excelente concordância com o cálculo variacional quântico que fornece para a carga atômica efetiva, $Z_{\mathrm{ef}}=Z-\frac{5}{16} \cdot{ }^{4}$ A aproximação de Bohr é excelente, pois $\frac{5}{16} \approx \frac{1}{4}$. É interessante comparar o resultado da energia quântica exata com o resultado calculado por Bohr. ${ }^{4}$ Essa comparação é feita na Tabela 1, indicando novamente a excelente previsão clássica feita por Bohr. 
Tabela 1. Energias (au) exatas e previstas pelo modelo de Bohr

\begin{tabular}{ccc}
\hline$Z$ & $E_{\text {exata }}$ & $E_{\text {Bohr }}$ \\
\hline 2 & $-2,9036$ & $-3,0625$ \\
3 & $-7,2800$ & $-7,5625$ \\
4 & $-13,6556$ & $-14,0625$ \\
5 & $-22,0310$ & $-22,5625$ \\
6 & $-32,4063$ & $-33,0625$ \\
\hline
\end{tabular}

\section{FORMAÇÃO DA MOLÉCULA DE $\mathrm{H}_{2}$}

No final da parte III, no item Formação de sistemas, Bohr discute qualitativamente a formação de moléculas. ${ }^{1,2}$ Niels Bohr não publica na trilogia um desenho feito a mão bastante ilustrativo, sobre a formação de diversas moléculas. Entretanto, essa figura foi feita no seu caderno de anotações ${ }^{1,2}$ e uma reprodução do original é apresentada na Figura 5. Bohr considera a formação de várias moléculas, $\mathrm{H}_{2}, \mathrm{H}_{2} \mathrm{O}$, $\mathrm{O}_{3}, \mathrm{CH}_{4}$ e $\mathrm{H}_{2} \mathrm{C}_{2}$, de acordo com seu modelo. A formação da molécula de $\mathrm{H}_{2}$, com base nos argumentos apresentado por Bohr, será discutida. Primeiro Bohr apresenta argumentos qualitativos para em seguida passar para o trabalho teórico.

Inicialmente são considerados dois átomos de hidrogênio afastados um do outro, como na Figura 6. Por meio de forças externas um núcleo se aproxima do outro. Bohr considera esse deslocamento dos núcleos tão lento que as órbitas individuais não são afetadas. Isso seria o equivalente a não ocorrer transições eletrônicas durante o processo de aproximação, coerente com a aproximação de Born-Oppenheimer. ${ }^{4}$ Para uma certa distância entre os dois núcleos os planos das órbitas se tornam coincidentes, formando assim a molécula diatômica. Uma ilustração dessa discussão qualitativa, e inspirada no texto de H.A. Kramers, ${ }^{5}$ é apresentada na Figura 6.

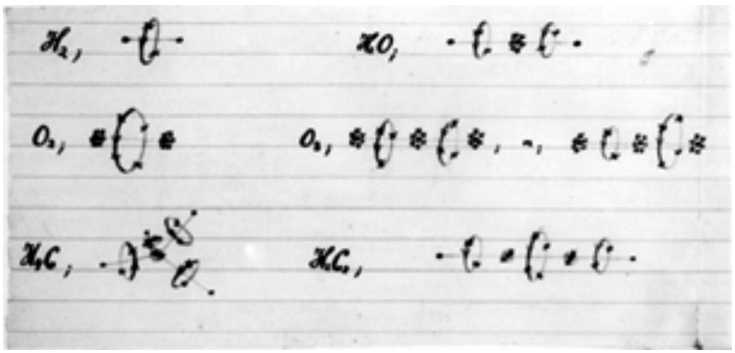

Figura 5. Figura do caderno de anotações do Bohr, gentilmente cedida pelo Arquivo Bohr, Copenhagen (a)<smiles>C1CC1</smiles>

(b)

(c)
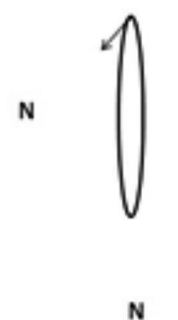

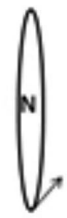

N ou seja,

\section{A MOLÉCULA DE BOHR}

A Figura 7 representa uma visão frontal da molécula de $\mathrm{H}_{2}$. Para que haja o equilíbrio, a força total deve ser nula. Três forças devem ser consideradas: a força atrativa dos elétrons com os núcleos, $\vec{F}_{\text {en }}$, a repulsiva entre os dois elétrons, $\vec{F}_{\text {ee }}$, a repulsiva entre os núcleos, $\vec{F}_{\text {nn }}$ e a centrífuga, $\vec{F}_{\mathrm{c}}$. Para o equilíbrio de forças na direção horizontal, considerando o ângulo $\theta$ como na Figura 7 tem-se,

$$
\begin{aligned}
& F_{\text {en, horizontal }}=\frac{e^{2}}{r^{2}} \cos \theta=\frac{e^{2} b}{r^{3}}=\frac{e^{2} b}{\left(a^{2}+b^{2}\right)^{3 / 2}} \\
& F_{\text {nn, horizontal }}=\frac{e^{2}}{(2 b)^{2}}=\frac{e^{2}}{4 b^{2}}
\end{aligned}
$$

Como são dois elétrons deve-se igualar $2 \times F_{\text {en, horizontal }}=F_{\text {nn, horizontal }}$,

$$
R=\frac{2 e^{2} b}{\left(a^{2}+b^{2}\right)^{3 / 2}}-\frac{e^{2}}{4 b^{2}}=0
$$

De forma análoga, para as três forças na direção vertical,

$$
\begin{aligned}
& F_{\text {en, vertical }}=\frac{e^{2}}{r^{2}} \sin \theta=\frac{e^{2} a}{r^{3}}=\frac{e^{2} a}{\left(a^{2}+b^{2}\right)^{3 / 2}} \\
& F_{\text {ee, vertical }}=\frac{e^{2}}{(2 a)^{2}}=\frac{e^{2}}{4 a^{2}} \\
& F_{\mathrm{c}}=\frac{m v^{2}}{a}
\end{aligned}
$$

O equilíbrio entre as forças será descrito por, $2 \times F_{\text {en, vertical }}-$ $F_{\text {ee, vertical }}=\frac{m v^{2}}{a}$ ou

$$
Q=\frac{2 e^{2} a}{\left(a^{2}+b^{2}\right)^{3 / 2}}-\frac{e^{2}}{4 a^{2}}-\frac{m v^{2}}{a}=0
$$

A notação aqui usada, $R$ e $Q$, é a mesma usada por Bohr. A procura da solução do sistema,

$$
\begin{aligned}
& R(a, b)=0 \\
& Q(a, b)=0
\end{aligned}
$$

foi o próximo passo dado por Bohr.

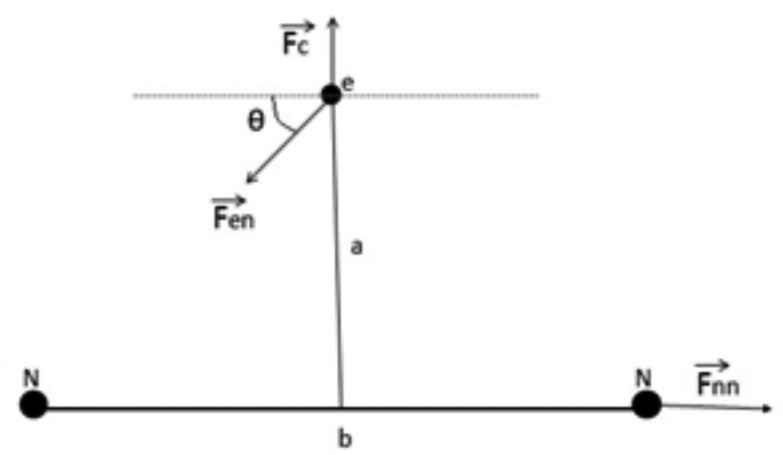

Figura 7. Diagrama de forças na molécula de $\mathrm{H}_{2}$

Bohr resolveu o sistema por substituição. De $R(a, b)=0$, isto é, $\frac{2 e^{2} b}{\left(a^{2}+b^{2}\right)^{3 / 2}}=\frac{e^{2}}{4 b^{2}}$ retira-se $8 b^{3}=\left(a^{2}+b^{2}\right)^{3 / 2}$. Tomando a potência $2 / 3$ dos dois lados pode-se desenvolver,

$$
\begin{aligned}
& 4 b^{2}=a^{2}+b^{2} \\
& 3 b^{2}=a^{2} \\
& a=\sqrt{3} b
\end{aligned}
$$

Figura 6. Formação da molécula de $\mathrm{H}_{2}$ 
O resultado $a=\sqrt{3} b$ deve ser substituído em $Q=0$ para se obter a solução geral. Usando, $\left(a^{2}+b^{2}\right)^{3 / 2}=\left(a^{2}+a^{2} / 3\right)^{3 / 2}=\left(4 a^{2} / 3\right)^{3 / 2}=$ $(4 / 3)^{3 / 2} a^{3}$ calcula-se,

$$
Q=\frac{2 e^{2} a}{(4 / 3)^{3 / 2} a^{3}}-\frac{e^{2}}{4 a^{2}}-\frac{m v^{2}}{a}=0
$$

ou após pequeno rearranjo,

$$
\frac{m v^{2}}{a}
$$

Por comparação com a equação (2) pode-se retirar o valor de $F$,

$$
\frac{2 e^{2} b}{\left(a^{2}+b^{2}\right)^{3 / 2}}=\frac{e^{2}}{4 b^{2}}
$$

como calculado por Bohr, no parágrafo 3 da parte III. ${ }^{1}$

\section{TESTE DO MODELO: ENERGIA DE DISSOCIAÇÃO DO H}

O modelo clássico de uma molécula diatômica estava construído, em completa analogia com o também modelo clássico atômico. Para um teste do modelo, Bohr calculou teoricamente a energia de dissociação da molécula de $\mathrm{H}_{2}$. Usando $E=-\frac{m F^{2} e^{4}}{2 n^{2} \hbar^{2}}$ fez a diferença da energia da molécula de $\mathrm{H}_{2}$ em relação a $2 \mathrm{H},{ }^{6}$

$$
E=-\frac{m F^{2} e^{4}}{2 n^{2} \hbar^{2}}
$$

pois $F=1$ para o átomo de hidrogênio. Com o valor já calculado, $E_{\mathrm{H}}=-0,5 \mathrm{au}$, calcula-se em unidades atômicas,

$$
D_{\mathrm{e}}=E\left(H_{2}\right)-2 E(H)=\left(F^{2}-1\right)
$$

Usando $F=1,049$, também já calculado, Bohr estabeleceu $D_{\text {e }}$ $=0,1001 \mathrm{au}, \mathrm{ou}$,

$$
D_{\mathrm{e}}=2,732 \mathrm{eV}
$$

Pelas equações (3) calcula-se o valor de $r=b$. Após substituição em $a=\sqrt{(3)} b$, retira-se a distância de equilíbrio, pois $R_{\mathrm{e}}=2 b$. O valor encontrado foi de $R_{\mathrm{e}}=1,1$ au ou $R_{\mathrm{e}}=0,5820 \AA$. Esses resultados são impressionantes para a época, como será discutido.

\section{COMPARAÇÃO COM CÁLCULOS QUÂNTICOS}

Os valores tabelados de energia de dissociação e distância de equilíbrio para o $\mathrm{H}_{2}$ são $D_{\mathrm{e}}=4,747 \mathrm{eV}$ e $R_{\mathrm{e}}=0,741 \AA$ e serão aqui usados como referência. ${ }^{7} \mathrm{O}$ resultado obtido por Bohr, se comparado com os dados tabelados, fornece erro percentual relativo de $42 \%$ para a energia. Para a posição de equilíbrio esse valor vale $22 \%$. Essa comparação pode parecer desanimadora, mas esse confronto não é prudente no momento. Uma análise dos primeiros resultados quânticos deve ser feita, antes de uma conclusão sobre o modelo.

$\mathrm{O}$ primeiro cálculo quântico para a molécula de $\mathrm{H}_{2}$ foi elaborado por Heitler e London em $1927 .{ }^{8}$ Para uma tradução para a língua inglesa desse artigo o leitor pode consultar o livro de Hettema. ${ }^{9}$ Esse trabalho de Heitler e London é considerado um clássico no contexto da química, pois inaugura a área da química quântica, em especial a teoria de ligação de valência. Após um longo cálculo de integrais para a solução da equação de Schrödinger, Heitler e London obtiveram $D_{\mathrm{e}}=3,140$ eV e $R_{\mathrm{e}}=0,869 \AA$. Portanto, esse dois resultados teóricos eram compatíveis com o modelo de Bohr e de certa forma indicavam que havia algo importante no modelo semi-clássico molecular de Niels Bohr.

Em contraste com a teoria de ligação de valência, o método dos orbitais moleculares foi também aplicado à molécula de $\mathrm{H}_{2}$, por Charles Coulson em 1937. ${ }^{10}$ Nesse cálculo Coulson encontrou $D_{\mathrm{e}}=$ $2,681 \mathrm{eV}$ e $R_{\mathrm{e}}=0,740 \AA$ A . Não havia praticamente erro na distância de equilíbrio, mas o valor da energia de dissociação era o mesmo (com dois algarismos significativos) do encontrado por Bohr. Um resumo da situação da molécula de Bohr e cálculos quânticos até 1937 é apresentado na Tabela 2.

Tabela 2. Modelo de Bohr, Heitler/London e Coulson para o $\mathrm{H}_{2}$

\begin{tabular}{lcc}
\hline Modelos teóricos & $D_{\mathrm{e}} / \mathrm{eV}$ & $R_{\mathrm{e}} / \AA$ \\
\hline Bohr, 1913 & 2,732 & 0,5820 \\
Heitler/London, 1927 & 3,140 & 0,869 \\
Coulson, 1937 & 2,681 & 0,740 \\
\hline
\end{tabular}

Os cálculos quânticos foram se aprimorando com novos métodos e novas tecnologias. O valor previsto pelo resultado quântico recente fornece os mesmos resultados tabelados. ${ }^{11}$ Entretanto, a descrição clássica da molécula de Bohr também sofreu avanços teóricos. Se a órbita dos átomos é não coincidente, como na Figura 6, e se as fases relativas dos elétrons são consideradas, ${ }^{11}$ a previsão teórica de molécula de Bohr modificada fornece $D_{\mathrm{e}}=4,490 \mathrm{eV}$ e $R_{\mathrm{e}}=0,714$ $\AA$. Esse resultado representa um erro de aproximadamente $5 \%$ na energia ou posição de equilíbrio.

\section{CONCLUSÕES}

A descrição teórica e clássica da molécula de $\mathrm{H}_{2}$ foi feita por Niels Bohr na sua célebre trilogia de 1913. Ao contrário do átomo de Bohr, o estudo molecular não é conhecido pelo estudante de química. O presente trabalho é uma tentativa de divulgar esse brilhante trabalho de Bohr.

A crença comumente aceita de que o modelo de Bohr não funcionava para átomo de dois elétrons deve ser abandonada no que diz respeito ao cálculo da energia. O erro para o átomo de hélio, no contexto de Bohr, vale 5\% e tende a cair com o aumento do número atômico. Para o íon $\mathrm{Li}^{+}$esse erro vale $4 \%$, decrescendo para $1 \% \operatorname{com} Z=10$. Essa tendência de queda no erro mostra que o modelo de Bohr se adapta melhor para um número atômico maior, pois nesse caso a perturbação da repulsão elétron-elétron se torna menos significativa.

O modelo molecular, o ponto principal do presente artigo, indica que este modelo oferece uma grande vantagem para o ensino, pois ilustra classicamente a formação da ligação química. Os resultados da energia de ligação e distância de equilíbrio são comparáveis em precisão com os cálculos quânticos da época, elaborados por Heitler e London, usando teoria de ligação de valência e por Coulson, usando a teoria dos orbitais moleculares. Da mesma forma que o modelo quântico foi refinado, o modelo molecular de Bohr pode também sofrer modificações, fornecendo resultados compatíveis com os cálculos quânticos atuais. $\mathrm{O}$ erro nesse modelo modificado de Bohr equivale a aproximadamente $5 \%$ se comparado com os resultados exatos.

\section{AGRADECIMENTOS}

Gostaríamos de agradecer ao $\mathrm{CNPq}$ pelo apoio financeiro e ao Niels Bohr Archive, Copenhagen, pela permissão para reproduzir as fotos e o desenho feito por Niels Bohr. 


\section{REFERÊNCIAS}

1. Bohr, N. Sobre a constituição de átomos e moléculas, Textos Fundamentais da Física Moderna, $2^{\text {nd }}$ ed., Fundação Calouste Gulbenkian: Lisboa, 1979, vol. 2.

2. Bohr, N. Em Niels Bohr: Collected Works; Rosenfeld, L.; Hoyer, U., eds.; North-Holland Publishing Company: Amsterdam, 1981, vol. 2.

3. Braga, J. P.; Filgueiras, C. A. L.; Quim. Nova 2013, (Submetido).

4. Braga, J. P.; Fundamentos de Química Quântica, Editora UFV: Viçosa, 2007.

5. Kramers, H.; The atom and the Bohr theory of its structure, an elementary presentation, Gyldendal: London, 1923.
6. Kragh, H.; J. Chem. Educ. 1977, 54, 208.

7. Herzberg, G.; Molecular Spectra and Molecular Structure, $2^{\text {nd }}$ ed.,Van Nostrand: Princeton, 1950.

8. Heitler, W.; London, F.; Zeitschrift für Physik 1927, 44, 455.

9. Heitler, W.; London, F. Em Quantum Chemistry: Classic Scientific Papers; Hettema, H., eds.; World Scientific: Singapore, 2000, vol. 8.

10. Coulson, C. A.; Trans. Faraday Soc. 1937, 33, 1479.

11. Svidzinsky, A.; Scully, M.; Herschbach, D.; Proceedings of the National Academy of Sciences 2005, 102, 11985. 